\title{
Measuring Surface Tensions of Soft Solids with Huge Contact-Angle Hysteresis
}

\author{
Jin Young Kim $\odot$, Stefanie Heyden, Dominic Gerber $\odot$, Nicolas Bain, Eric R. Dufresne๑, and Robert W. Style ${ }^{*}$ \\ Department of Materials, ETH Zürich 8093, Switzerland
}

(Received 8 March 2021; revised 20 April 2021; accepted 19 May 2021; published 7 July 2021)

\begin{abstract}
The equilibrium contact angle of a droplet resting on a solid substrate can reveal essential properties of the solid's surface. However, when the motion of a droplet on a surface shows significant hysteresis, it is generally accepted that the solid's equilibrium properties cannot be determined. Here, we describe a method to measure surface tensions of soft solids with strong wetting hysteresis. With independent knowledge of the surface tension of the wetting fluid and the linear-elastic response of the solid, the solid deformations under the contact line and the contact angle of a single droplet together reveal the difference in surface tension of the solid against the liquid and vapor phases If the solid's elastic properties are unknown, then this surface tension difference can be determined from the change in substrate deformations with contact angle. These results reveal an alternate equilibrium contact angle, equivalent to the classic form of Young-Dupré, but with surface tensions in place of surface energies. We motivate and apply this approach with experiments on gelatin, a common hydrogel.
\end{abstract}

DOI: $10.1103 /$ PhysRevX.11.031004

Subject Areas: Fluid Dynamics, Mechanics, Soft Matter

\section{INTRODUCTION}

The simplest and most widely used technique to characterize a solid surface is the measurement of the contact angle of sessile droplets. This is typically achieved by relating the equilibrium contact angle $\theta_{\text {eq }}$ to the surface-energy difference across a contact line via the law of Young-Dupré:

$$
\gamma_{l v} \cos \theta_{\mathrm{eq}}=\gamma_{s v}-\gamma_{s l} .
$$

Here, $\gamma$ represents surface energy, and subscripts $s, v, l$ correspond to solid, vapor, and liquid, respectively. Absolute values of surface energies can be estimated by using information from wetting experiments with multiple different liquids (see, e.g., Ref. [1]). On many surfaces, however, it is impossible to accurately measure equilibrium contact angles: the contact angle exhibits hysteresis due, for example, to surface roughness, pinning, or surface adaption [2]. Then, only advancing and receding contact angles can be measured reliably [3]. Hysteresis-free surfaces are rare in the lab, and even rarer in real-world applications. Thus, quantitative approaches are needed to characterize the surface properties of hysteretic surfaces.

This overarching challenge is reflected in the more specific case of wetting on soft surfaces. In such systems, contact lines drive significant deformation of solid

\footnotetext{
*robert.style@mat.ethz.ch
}

Published by the American Physical Society under the terms of the Creative Commons Attribution 4.0 International license. Further distribution of this work must maintain attribution to the author(s) and the published article's title, journal citation, and DOI. substrates, typically a swollen polymer network. Relevant materials include hydrogels, silicones, organogels, ionogels, rubbers, and thermoplastic elastomers. In general, such surfaces are hysteretic. However, with few exceptions [4,5], the study of soft wetting has focused on silicone gels and elastomers, using immiscible liquids such as water or glycerol (see, e.g., Refs. [6-10]). These show little to no hysteresis [11], and thus avoid the problems inherent in working with "nonideal" surfaces.

Hydrogels are a very important class of soft solids. They can take a variety of forms, and are widespread due to their central role in biological tissue. Their wetting properties are rich but difficult to measure quantitatively. For example, it may seem natural that water should spread on hydrogels, since they are made mostly of the same liquid. However, water droplets often take finite contact angles on hydrogel surfaces [12], and can exhibit large contact-angle hysteresis [13]. Hydrogel surfaces can also adapt to their environment, redistributing the network $[14,15]$ and reorienting its subunits [16], which give rise to dynamic surface properties. Physically cross-linked hydrogels, typically found in or derived from living systems, can undergo time-dependent changes in the network structure [17]. Finally, hydrogels can dry out, swell, and even dissolve during wetting [18].

Here, we introduce a method to measure surface tension differences across contact lines of hysteretic soft materials, based on the measurement of microscopic deformations of the gel upon wetting. Essentially, this is achieved by measuring $\theta_{0}$, the angle at which the surface tension forces acting at the contact line are in horizontal force balance. This approach requires knowledge of the liquid-vapor surface tension of the wetting liquid, but the elastic constants of the gel can be unknown. We demonstrate this 

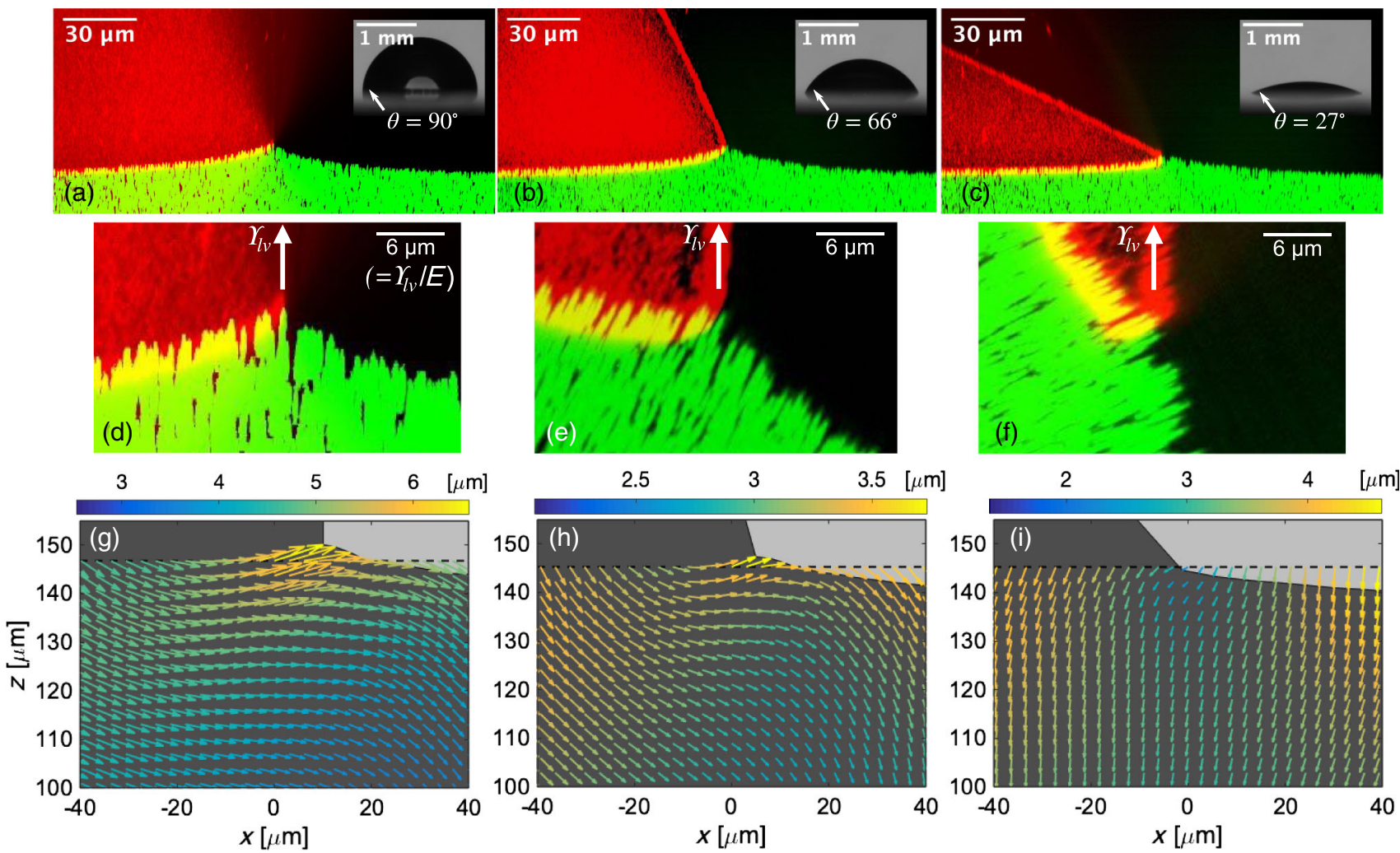

FIG. 1. Sessile glycerol-water droplets on gelatin gels swollen with the same liquid. A droplet is deposited on gelatin, where its contact line pins so that liquid can be removed without moving the contact line. (a)-(c) Side views of contact lines with different contact angles $\left(90^{\circ}, 66^{\circ}, 27^{\circ}\right.$, respectively) imaged with macro photography (inset) and confocal microscopy, where red (green) nanoparticles label the droplets (gelatin), respectively. (d)-(f) Enlargement of the wetting ridges in (a)-(c), rotated so that the liquid-vapor interface is vertical. Panels (g)-(i) show the displacements under the ridge, calculated by tracking nanoparticle displacements between a relaxed state where the surface is flooded with liquid and the deformed state under a contact line. The arrow colors indicate their magnitude.

approach with gelatin, a ubiquitous biological gel which exhibits huge contact-angle hysteresis. The measured value of the surface-tension difference across the contact line is very similar to the liquid-vapor surface tension of the gelatin solution just before gelation. This suggests that the surface tension of the interface between a gel and a droplet of its solvent can be negligible.

\section{CHARACTERISING GEL DEFORMATIONS UNDER A CONTACT LINE}

For these experiments, we use a soft gelatin gel consisting of $5 \mathrm{~g}$ of gelatin per $100 \mathrm{ml}$ of solvent phase, which is 4 parts glycerol to 1 part de-ionized water by volume. This particular ratio was chosen, as it is essentially the same as a hydrogel, but shows minimal evaporation or hygroscopic behavior during experiments, avoiding the need for humidity control [19]. The gel has Young's modulus, $E=11.0 \pm 1.5 \mathrm{kPa}$, Poisson ratio $\nu=0.39 \pm 0.05$, and poroelastic diffusivity $D=3.0 \pm 1.5 \times 10^{-9} \mathrm{~m}^{2} / \mathrm{s}$, as measured by indentation [20]. When we place a droplet of the solvent phase (surface tension $64 \mathrm{mN} / \mathrm{m}$ ) on the surface of the cured gel, the droplet advances with a contact angle of $\theta \sim 130^{\circ}$. When liquid is subsequently removed with a pipette, the contact line remains pinned as $\theta \rightarrow 0$ (see insets in Fig. 1). This enormous contact angle hysteresis allows us to create stable contact lines with a wide range of $\theta$. In every experiment, we use fresh, neverwetted gelatin surfaces, so the surface outside the droplet's contact line has never been in contact with bulk liquid.

We ensure that there is sufficient time for the droplet to equilibrate with the substrate, and with the surrounding atmosphere, by leaving it for $t_{\mathrm{eq}} \approx 10^{3} \mathrm{~s}$ before imaging. This allows the gelatin to relax poroelastically over a region of size $\sqrt{D t_{\mathrm{eq}}} \approx 1 \mathrm{~mm}$ from the contact line. This is much larger than the film thickness and the field of view under the contact line that we use during imaging, so there should be minimal residual poroelastic stresses to affect our results [21].

The surface tension of the droplet strongly deforms the underlying material. We place large, millimetric 4:1 glycerol:water droplets on a thin film (147 $\mu$ m thick) of gelatin coated on a glass slide. The films contain $0.003 \%$ by volume of embedded, fluorescent, 100-nm-diameter nanoparticles. These do not segregate to the film surface, and are so dilute that we do not expect their presence to affect the film's properties. $200 \mathrm{~nm}$ nanoparticles with a different emission spectrum are also attached to the glass slide, to act 
as reference points, and dispersed in the droplet. We image the sample from underneath with a $60 \times, 1.2 \mathrm{NA}$, waterimmersion objective on a confocal microscope (further details in Appendix A). This gives a 3D intensity map of the substrate and the droplet. We visualize the contact line by taking a maximum-intensity projection along a $30-\mu \mathrm{m}-$ long, straight, pinning-defect-free section of contact line, which collapses the 3D map into a side view of the contact line, as shown in Figs. 1(a)-1(c). Here, particles in the droplet are shown as red, while the those in the substrate are green. Note that the liquid is identical in both the droplet and gel phases. The images clearly show the wetting ridge that is pulled up by the surface tension of the droplet $\Upsilon_{l v}$. As $\theta$ reduces from $\theta=90^{\circ}$ to $27^{\circ}$, the shape of the wetting ridge changes.

What can we extract from the shapes of the ridges? In previous work on hysteresis-free silicone gels, the angles between the three interfaces where they meet at the contact line were found to be fixed [9]. This was interpreted as being due to a force balance between the surface tensions of the interfaces: $\Upsilon_{l v}, \Upsilon_{s v}$, and $\Upsilon_{s l}$. Then $\Upsilon_{s v}$ and $\Upsilon_{s l}$ could be extracted after measuring $\Upsilon_{l v}$ with standard techniques, and by applying the Neumann triangle construction [9]. This force balance is expected to hold only in a region around the contact line of size $\ll \Upsilon_{l v} / E$, the elastocapillary length. It is essential to distinguish surface energies $\gamma$ from surface tensions (or surface stresses) $\Upsilon$. The former represents the excess free energy of a material at its interface. The latter are the measurable two-dimensional stresses localized at the interface. In simple liquids (such as the droplet here), $\gamma_{l v}=$ $\Upsilon_{l v}$ [22]. However, for solids, $\gamma_{s}$ and $\Upsilon_{s}$ need not be the same, especially if $\gamma_{s}$ changes as the surface stretches [22-24]. In general, experiments that measure forces and displacements give access to surface tensions, as is the case here.

On gelatin, the microscopic geometry near the contact line appears to vary with $\theta$. Figures 1(d)-1(f) show enlarged images of the tips of the wetting ridges from Figs. 1(a)-1(c), rotated so that the liquid-vapor interface is vertical. The liquid-wedge angle seems to shrink dramatically as $\theta$ reduces. If this represented changes in Neumann's triangle, this could mean that $\Upsilon_{s v}$ and $\Upsilon_{s l}$ are changing close to the contact line for different values of $\theta$. On the other hand, the elastocapillary length, $\Upsilon_{l v} / E$, is only $6 \mu \mathrm{m}$-given the resolution of images in Fig. 1(d)-1(f), one should apply Neumann's triangle reluctantly.

Instead of focusing on the wetting ridge, we consider deformations away from the contact line, where strains are small and linear elasticity accurately captures the local balance of stress and strain. To access the stress-free locations of the particles, we flood the surface of the gel with the droplet phase at the end of the experiment. We track displacements of embedded nanoparticles as $\theta$ changes, using a modified version of the tracker described in Ref. [25]. Displacements are essentially zero in the direction parallel to the contact line. Thus, we collapse the displacements along the contact line to give 2D displacements maps, as shown in Figs. 1(g)-1(i). There, the arrows start at (interpolated) nanoparticle positions ( $x$, $z$ ) in the stress-free configuration, and end at the corresponding positions in the deformed state. Displacements decay to zero at the rigid gelatin-glass interface at $z=0$, as shown in the full displacement fields in the Supplemental Material [26].

The displacement maps immediately reveal large differences under the contact line for the various $\theta$. Most obviously, the magnitude of the displacements reduce significantly as the contact angle shrinks. Surprisingly, for $\theta=90^{\circ}$ and $66^{\circ}$, despite the fact that the liquid-vapor interface pulls upward, or to the left, there are strong displacements to the right underneath the contact line. For $\theta=27^{\circ}$, in-plane displacements reverse sign, and the contact line is pulled to the left. Interestingly, this would suggest that there is an intermediate angle where the average horizontal displacement vanishes-potentially identifying a point where none of the horizontal component of $\Upsilon_{l v}$ is transmitted to the underlying substrate. Additionally, there is also a small overall shrinkage of the substrate between the deformed and reference states, as seen clearly in Fig. 1(i). There, all displacements point slightly downward, despite $\Upsilon_{l v}$ pulling up on the ridge. This indicates that the flooding liquid slightly swells the film, suggesting a slight composition difference between the droplet and gel's solvent (which had time to equilibrate with the surrounding atmosphere). Equivalently, there is a uniform gel pore pressure $P$ that may vary between images.

\section{MEASURING SURFACE TENSIONS FROM SUBSTRATE DISPLACEMENT FIELDS}

The displacement field gives us access to the stresses acting on the surface of the gel. To characterize these, one option would be to convert displacements to strains, and then use the gel's poroelastic constitutive behavior to calculate the stress distribution in the substrate. However, this requires a knowledge of $P$, which is not easy to measure.

Instead, we can exploit the symmetries of the problem to cleanly visualize the magnitude and orientation of the surface forces. Figure 2(a) shows a schematic of the forces acting near the contact line. Locally, there is a region where the surface is deformed, with a size of $\mathcal{O}\left(\Upsilon_{l v} / E=6 \mu \mathrm{m}\right)$. We zoom out to look from a much larger scale - that of the film thickness, $h \gg \Upsilon_{l v} / E$, as shown in Fig. 2(b). Provided the droplet's contact radius is $\gg h$, the substrate appears as a flat, linear elastic film, being acted on by vertical and horizontal linear forces at the contact line:

$$
\Upsilon_{\perp}=\Upsilon_{l v} \sin \theta
$$

and

$$
\Upsilon_{\|}=\Upsilon_{s v}-\Upsilon_{s l}-\Upsilon_{l v} \cos \theta \equiv \Delta \Upsilon-\Upsilon_{l v} \cos \theta
$$




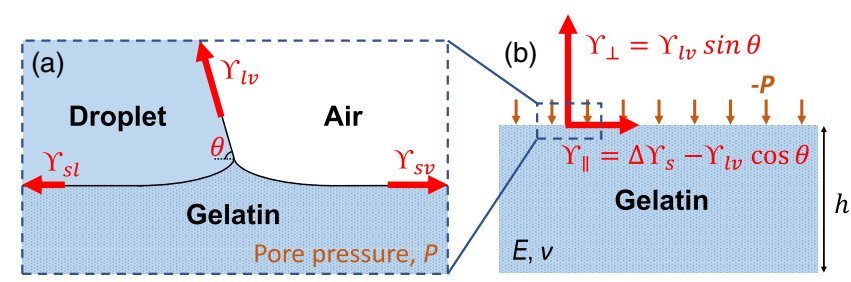

FIG. 2. A schematic diagram showing the forces acting on the gelatin. (a) Near the contact line, there is a highly deformed wetting ridge. The gelatin may also be deformed by a pore pressure $P$ that acts to swell or deswell the gelatin. (b) At a scale much bigger than that of the wetting ridge, the surface forces and pore pressure effectively act as horizontal and vertical line forces at the contact line, and as a uniform compressive pressure on the surface of a flat film.

respectively, while the presence of a pore pressure is equivalent to a uniform vertical compression on the surface with a pressure $-P$.

To isolate the effects of $\Upsilon_{\perp}$ and $\Upsilon_{\|}$, we note that $P$ affects only vertical displacements of the substrate, $u_{z}(x, z)$, as it is uniform across the substrate surface, and acts directly downward. Thus, we focus only on the horizontal displacements, $u_{x}(x, z)$. Furthermore, we notice that $\Upsilon_{\perp}$ results in horizontal displacements that are symmetric about the position of the contact line $(x=0)$, while $\Upsilon_{\|}$ results in antisymmetric horizontal displacements about $x=0$ (see the Appendix B for a proof). Thus, if we decompose $u_{x}$ into odd and even parts $u_{x}^{o}=\left[u_{x}(x, z)-\right.$ $\left.u_{x}(-x, z)\right] / 2$ and $u_{x}^{e}=\left[u_{x}(x, z)+u_{x}(-x, z)\right] / 2$, respectively, the resulting displacements take the form

$$
\begin{aligned}
& u_{x}^{o}=\frac{\Upsilon_{\perp}}{E} U_{\perp}(x, z, \nu), \\
& u_{x}^{e}=\frac{\Upsilon_{\|}}{E} U_{\|}(x, z, \nu),
\end{aligned}
$$

where the functions $U_{\perp}$ and $U_{\|}$are given in the Supplemental Material [26] (following Ref. [27]). Thus, if the experimentally measured $u_{x}^{o}$ and $u_{x}^{e}$ match the patterns of $U_{\perp}$ and $U_{\|}$, we can use their relative magnitudes to extract $\Upsilon_{\perp}$ and $\Upsilon_{\|}$. Note, however, that we expect the patterns to deviate in a region around the contact line of size $\mathcal{O}\left(\Upsilon_{l v} / E\right)$, due to contributions from solid surface tension [22].

Indeed, the decomposed displacement fields match well with linear-elastic predictions for $U_{\|, \perp}$. Figure 3 shows $u_{x}^{o}$ [Figs. 3(a)-3(c)] and $u_{x}^{e}$ [Figs. 3(e)-3(g)] for the different $\theta$ in Fig. 1, while Figs. 3(d) and 3(h) show $U_{\perp}$ and $U_{\|}$. These show strong qualitative agreement, including $u_{x}^{e}$ for $\theta=27^{\circ}$, where the sign of the displacements is reversed.
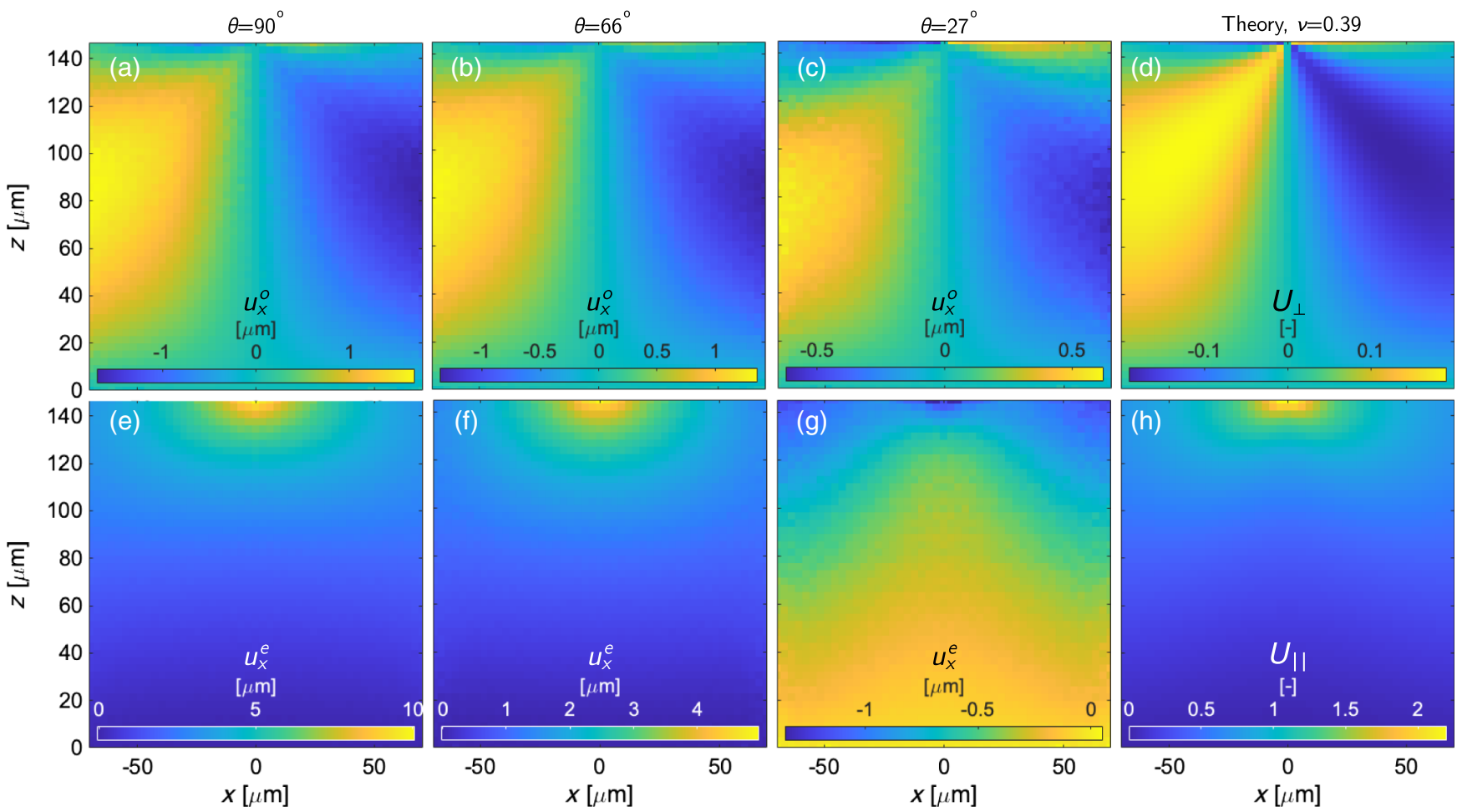

FIG. 3. Decomposing the horizontal displacements under a contact line. The top (bottom) rows show the odd (even) parts of $u_{x}(x, z)$ : $u_{x}^{o}$ and $u_{x}^{e}$. The first three columns (a)-(c),(e)-(g) correspond to $\theta=90^{\circ}, 66^{\circ}, 27^{\circ}$, respectively. The final column (d),(h) shows $U_{\perp}$ and $U_{\|}$from Eq. (4), using $\nu=0.39$ and $h=147 \mu \mathrm{m}$. The experiments show good agreement with the theory. Note that the magnitude of (g) is negative, causing the colors to be the reverse of those in (e), (f), and (h). 
We extract $\Upsilon_{\perp}$ and $\Upsilon_{\|}$using a least-squares fitting of the odd and even displacement fields to the theoretical expressions [Eqs. (4) and (5)]. We use the entire image for fitting, as this gives essentially the same results as when we ignore the region close to the contact line (e.g., within a distance $3 \Upsilon_{l v} / E$ ). Images showing residual errors between the data and the theory are given in the Supplemental Material for each dataset [26]. The resulting values of $\Upsilon_{\perp}$ and $\Upsilon_{\|}$are shown in Figs. 4(a) and 4(b). The error bars reflect the measured uncertainty in $E$ and $\nu$. Note that these are much larger for $\Upsilon_{\perp}$ than $\Upsilon_{\|}$. Measurement of $\Upsilon_{\perp}$ is much more sensitive to the value of $\nu$ (which has relatively wide error bars), as it involves substrate contractions perpendicular to the loading direction.

Furthermore, the fitted values of $\Upsilon_{\perp}$ and $\Upsilon_{\|}$agree well with the expected forms given in Eqs. (2) and (3). Using the value of $\Upsilon_{l v}=64 \mathrm{mN} / \mathrm{m}$, measured by hanging drop tensiometry, we plot these equations as dashed lines in Figs. 4(a) and 4(b). For $\Upsilon_{\perp}$, we see agreement with the data with no fitting parameters. For $\Upsilon_{\|}$, there is one fitting parameter, $\Delta \Upsilon$, the difference in the solid-vapor and solidliquid surface tensions. For these data, we find $\Delta \Upsilon=$ $51 \pm 3 \mathrm{mN} / \mathrm{m}$. Note that $\Delta \Upsilon$ could also be extracted with data from a single contact angle $\theta$ using this approach.

When the linear elastic properties of the gel $(E$ and $\nu)$ are unknown, a series of measurements with different contact angles can still reveal $\Delta \Upsilon$. The key is the dependence of the magnitude of $u_{x}^{e}$ on the contact angle $\theta$. At some angle, $\theta_{0}$,
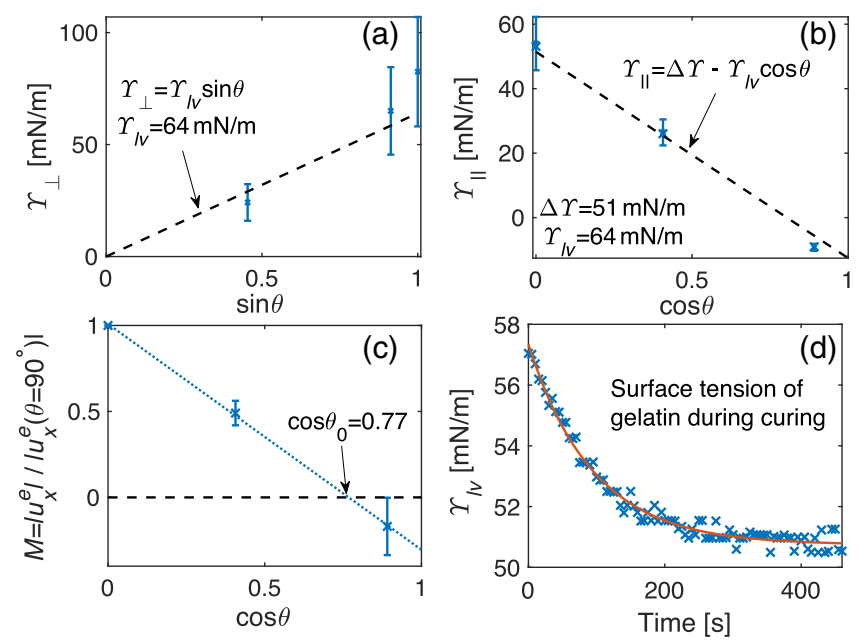

FIG. 4. Measuring surface tensions on the gel. Panels (a) and (b) show $\Upsilon_{\perp}(\theta)$ and $\Upsilon_{\|}(\theta)$, measured by fitting displacement fields to the theoretical expressions in Eqs. (4) and (5). The dashed line shows the theoretical predictions from Eqs. (2) and (3). Panel (c) shows $M$, the relative magnitudes of the displacement fields in Figs. $3(\mathrm{e})-3(\mathrm{~g})$. The angle $\theta_{0}$, where $M=0$, corresponds to the point where the stresses acting at the gel's surface balance horizontally. (d) The surface tension of a curing gelatin droplet, measured via pendant-droplet tensiometry. The red curve shows an exponential fit to the data, which plateaus to a value of $51 \mathrm{mN} / \mathrm{m}$. $u_{x}^{e}=0$. At this point, $\Upsilon_{\|}=0$, and $\Delta \Upsilon=\Upsilon_{l v} \cos \theta_{0}$, as given by Eq. (3). We determine $\theta_{0}$ by plotting the relative magnitude $M$ of $u_{x}^{e}(\theta)$ and $u_{x}^{e}\left(\theta=90^{\circ}\right)$ against $\cos \theta$ in Fig. 4(c). $M$ is defined as the constant that minimizes $\left[u_{x}^{e}\left(\theta=90^{\circ}\right)-M u_{x}^{e}(\theta)\right]^{2}$ over all the pixels in Fig. 3. Then we fit a line $M=1-\alpha \cos \theta$ to the data, and extract $\cos \theta_{0}=1 / \alpha=0.77$. This yields $\Delta \Upsilon=49 \pm 5 \mathrm{mN} / \mathrm{m}$, which is in good agreement with the values that we extracted from the mechanical model using the elastic properties of the gel.

To corroborate these results, we measured the surface tension of gelatin solutions as they cured using pendantdrop tensiometry [28]. Gelatin, dissolved at high temperature, does not instantly cure upon cooling to room temperature. Instead it takes $\mathcal{O}(20 \mathrm{~min})$ to gel [29]. Thus, we can hang a liquid droplet of curing gelatin at room temperature, and measure its surface tension as it cross-links, as shown in Fig. 4(d). Note that this method, which assumes hydrostatic equilibrium, is only applicable as long as the gelatin remains fluid. Therefore, it can reliably report surface tension on the approach to the gel point, but not past it. As gelation proceeds, the surface tension reduces. After around $5 \mathrm{~min}$, the apparent surface tension plateaus to a final value of $51 \pm 1 \mathrm{mN} / \mathrm{m}$. With the assumption that the surface is not significantly altered across the gel point, this suggests that $\Upsilon_{s v} \approx 51 \mathrm{mN} / \mathrm{m}$. In the gel state, we further anticipate that $\Upsilon_{s l} \ll \Upsilon_{s v}$, as both sides of the liquid-gel interface are predominantly the same solvent, and the concentration of the polymer is very dilute, only $3 \%-4 \%$. Together, these suggest that $\Delta \Upsilon=51 \pm 1 \mathrm{mN} / \mathrm{m}$, in excellent agreement with our gel deformation measurements.

At the angle $\theta_{0}$, the liquid-vapor interface transmits no in-plane forces to the substrate, $\Upsilon_{\|}=0$. According to Eq. (3), this mechanical equilibrium is given by

$$
\Upsilon_{l v} \cos \theta_{o}=\Upsilon_{s v}-\Upsilon_{s l}
$$

It is independent of the bulk mechanical properties, such as $E$ and $\nu$, and the far-field boundary conditions such as the film thickness $h$. This is similar in form to the law of YoungDupré, Eq. (1). While $\theta_{\mathrm{eq}}$ reveals surface energies, $\theta_{0}$ reveals surface tensions localized to the contact line. These two are equivalent $\left(\theta_{0}=\theta_{\text {eq }}\right)$ when $\Upsilon_{s v}-\Upsilon_{s l}=\gamma_{s v}-\gamma_{s l}$. This is the typical assumption for gels, since they are mostly made of a simple liquids [30-34]. However, any solid material can, in principle, have different values of $\gamma$ and $\Upsilon$ [35]. The relevance of this distinction for swollen polymer networks remains an actively debated topic.

\section{A. Depinning forces}

Our results allow us to calculate the maximum force that can be exerted on the substrate before depinning occurssomething that cannot be measured without an accurate value of $\Delta \Upsilon$. Consider a droplet at its advancing contact angle $\theta_{a}$, as shown in Fig. 5. Evaluating Eqs. (2) and (3) in 


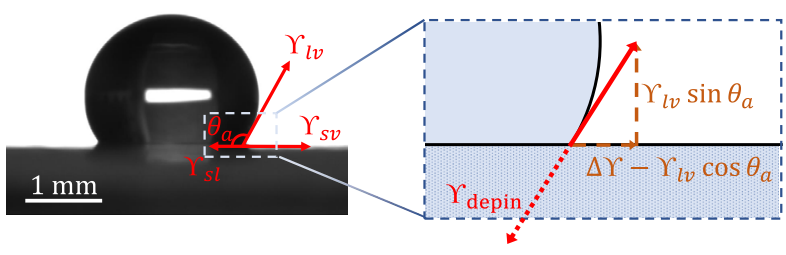

FIG. 5. The depinning force at the contact line at the advancing contact angle. This opposes the surface-tension forces from $\Upsilon_{l v}$, $\Upsilon_{s v}$, and $\Upsilon_{s l}$. Note that, in general, this is not parallel to the liquid surface at the contact line.

this configuration yields the total magnitude of the depinning force:

$$
\Upsilon_{\text {depin }}=\sqrt{\Upsilon_{l v}^{2}+(\Delta \Upsilon)^{2}-2 \Upsilon_{l v} \Delta \Upsilon \cos \theta_{a}}
$$

For the current experiments, this gives $\Upsilon_{\text {depin }}=$ $104 \mathrm{mN} / \mathrm{m}$. It is interesting to speculate what sets this value, as hysteresis can arise for multiple reasons, including surface roughness, microscopic chemical heterogeneity of the surface, molecular reorientation at the surface, and dynamic hysteresis due to viscoelastic or poroelastic braking [36]. Here, it is unlikely that there is significant surface roughness of the native gel surface, as surface tension keeps it extremely flat during the curing process. Viscoelastic braking $[6,37]$ or poroelastic braking [38] is also unlikely here, as droplet contact angles show little relaxation over the course of days. Instead, we expect it to be related to processes at the surface including yielding and damage $[39,40]$, molecular reorientation of the surface $[13,16,41,42]$, or potentially straindependent surface properties $[43,44]$. Fully understanding the origins of pinning here is beyond the scope of this paper. However, we believe our approach provides important new tools to explore this question.

\section{CONCLUSIONS}

In summary, we present a new approach for the characterization of surface properties by wetting experiments. Traditionally, the contact angle of the droplet is interpreted to reveal interfacial free-energy differences. While this is on the firmest conceptual foundations, practically the equilibrium contact angle can only be narrowed down within a range set by the advancing and receding contact angles. On all but ideal surfaces, this range is too broad to pin down surface properties. We argue for a different approach, based on the less stringent requirement of mechanical, rather than thermodynamic, equilibrium. Specifically, we have shown that it is possible to determine the contact angle where the net in-plane forces on the substrate is zero. In this way, we can quantify differences in interfacial tension, even in the presence of large contact-angle hysteresis. This generalizes the concept of the equilibrium contact angle, and is captured in compact form by Eq. (6), which echoes the original formulation of Young and Dupré.
Our approach enables the measurement of surfacetension differences and depinning forces at contact lines on soft solids, even in the presence of huge contact-angle hysteresis. Even when the solid's elastic properties are unknown. As we expect that the surface tension between a gel and its own solvent $\left(\Upsilon_{s l}\right)$ is minimal, this effectively allows us to evaluate absolute values of $\Upsilon_{s v}$. Our approach can be used on relatively stiff substrates: potentially up to $\mathcal{O}(100 \mathrm{kPa})$, when using light microscopy to track displacements. This is in contrast to elastocapillary approaches limited to softer materials [9,30,31,45,46]. Additionally, our technique does not restrict one to immiscible substrateliquid pairings (cf. Refs. [9,47,48]), and the measured surface stress values should be for the fully relaxed state, unlike other options, which extract surface stress values in situations where the surface is significantly deformed $[9,11,30,49]$. Although we have used the entire displacement field under a contact line, similar information should be measurable from the displacements at the surface away from the contact line. Further, here, we only consider the common case where a droplet is much larger than the substrate layer thickness, but we note that smaller probe droplets can certainly be used by adapting the theory presented above to use previous analytic solutions [50].

We anticipate that future work can extract further information about the system from the near-contact-line region by comparison with analytic models that account for different types of behavior at the gel surface [51-53]. Our technique could also be used to measure mechanical substrate properties with one single measurement (cf. Ref. [54] for alternative approaches), as vertical displacements of the substrate contain information about the pore pressure in the film, while the detailed pattern of substrate deformations depends on $\nu$ and $E$. Importantly, it is well known that surface properties of soft materials can be history dependent. Thus, we anticipate that measured value of surface tensions, and the contact angle representing mechanical equilibrium, may change for advancing and receding contact lines. For such studies, systematic variation of liquid-substrate combinations will be essential.

We have archived our tracking code with a permanent DOI [55].

\section{ACKNOWLEDGMENTS}

R.W. S. is supported by the Swiss National Science foundation (Grant No. 200021-172827). J. Y. K. is supported by the MOTIE in Korea, under the Fostering Global Talents for Innovative Growth Program supervised by the KIAT (Grant No. P0008746). We acknowledge helpful conversations with Katrina-Smith Mannschott and Anand Jagota.

\section{APPENDIX A: MATERIALS AND METHODS}

To make the gelatin gels, we dissolved $5 \mathrm{~g}$ gelatin (general purpose grade gelatine, Fisher) in a mixture of $80 \mathrm{ml}$ glycerol (VWR) and $20 \mathrm{ml}$ de-ionized water. 
The mixture was heated with stirring at $90^{\circ}$ for $30 \mathrm{~min}$ (following Ref. [39]). After heating, $100 \mu \mathrm{l}$ of 100-nm-diameter, carboxylate-modified, red fluorescent nanoparticles $(2 \%$ solids, Fluospheres, ThermoFisher Scientific) were added into the mixture, and it was poured into molds and allowed to cool for $24 \mathrm{~h}$ at $4{ }^{\circ} \mathrm{C}$ before being used. For measuring mechanical properties, we filled $1-\mathrm{cm}-$ deep petri dishes with the gelatin.

For confocal imaging, we created thin films of gelatin on $50 \mathrm{~mm}$ petri dishes with a no. 1.5 glass cover slip as their bottom surface (P50G-1.5-30-F, MatTek). This involved first attaching yellow-green fluorescent nanoparticles to the glass surface, which act as fixed reference points for tracking the bottom of the gelatin layer, and then adding the gelatin layer. To attach the reference particles to the glass surface, we follow the protocol described in Ref. [56]. In brief, we activate the surface in a UV-ozone cleaner, functionalize it via vapor deposition of (3-Aminopropyl)triethoxysilane, and then submerge it in a solution containing 200-nm-diameter, yellow-green, carboxylate-modified, fluorescent nanoparticles (2\% solids, Fluospheres, ThermoFisher). We then rinse this with de-ionized water. To make the thin films of gelatin, we placed $30 \mu \mathrm{l}$ of the hot gelatin solution on the bead-coated glass, and then covered this with a $18-\mathrm{mm}-$ diameter, no. 1.5 circular cover glass (Paul Marienfeld, $\mathrm{GmbH}$ ). After curing, the cover glass was gently removed with tweezers, and the substrate was used for experiments.

For imaging of contact lines, we make a solution containing $8 \mathrm{ml}$ glycerol, $2 \mathrm{ml}$ de-ionized water, and $10 \mu \mathrm{l}$ of the (2\% solids) yellow-green nanoparticles. Then we place a $5 \mu \mathrm{l}$ droplet of the solution on the gelatin layer. We reduced the size of the droplet when required by manually removing liquid with a pipette, approximately $1 \mu \mathrm{l}$ at a time. After each change in droplet volume, we let it rest for at least $20 \mathrm{~min}$ before imaging.

3D confocal imaging was done on a microscope (Nikon, Eclipse Ti2) with a spinning disk confocal system (Yokagawa CSU-X1) using a 60× water immersion objective lens (Nikon, MRD07602). We used 488 and $560 \mathrm{~nm}$ lasers for yellow-green and red fluorescent nanoparticles, respectively. Images were acquired with at $330 \mathrm{~nm}$ intervals in $z$ to obtain 3D measurements.

We measure the surface tension of liquids using a homemade pendant-droplet tensiometry setup. Droplet shapes are analyzed with axisymmetric drop shape analysis [28]. For the droplet phase, we measure $\Upsilon_{l v}$ of the pure liquid, and liquid that has been allowed to equilibrate on the gelatin substrate for an hour. This gives $\Upsilon_{l v}=66 \pm 1$ and $64 \pm 1 \mathrm{mN} / \mathrm{m}$, respectively, suggesting that there is very little contamination of the droplet by material from the gelatin.

\section{APPENDIX B: ODD AND EVEN RESPONSES TO LINE LOADINGS}

For a vertical line force $\Upsilon_{\perp}$ acting on the substrate as shown in Fig. 2(b), there is complete left-right symmetry to the problem. This immediately implies symmetric inward or outward displacements, so that the resulting displacement field $u_{x}^{\perp}(x, z)$ is odd.

For a horizontal line force $\Upsilon_{\|}$, let the solution be $u_{x}^{\|}\left(x, z, \Upsilon_{\|}\right)$. The solution for $\Upsilon_{\|} \rightarrow-\Upsilon_{\|}$satisfies $u_{x}^{\|}\left(x, z, \Upsilon_{\|}\right)+u_{x}^{\|}\left(x, z,-\Upsilon_{\|}\right)=0$, as the sum of the line forces $\Upsilon_{\|}$and $-\Upsilon_{\|}$are zero, so the sum of the resulting displacement fields must also be zero. Furthermore, the solution for a negative line force is the same as the solution for a positive line force, reflected about $x=0$. Mathematically this can be expressed as $u_{x}^{\|}\left(x, z,-\Upsilon_{\|}\right)=-u_{x}^{\|}\left(-x, z, \Upsilon_{\|}\right)$. Combining these two equations gives $u_{x}^{\|}\left(x, z, \Upsilon_{\|}\right)=u_{x}^{\|}\left(-x, z, \Upsilon_{\|}\right)$, so $u_{x}^{\|}$is even.

[1] D. K. Owens and R. Wendt, Estimation of the Surface Free Energy of Polymers, J. Appl. Polym. Sci. 13, 1741 (1969).

[2] P.-G. de Gennes, F. Brochard-Wyart, and D. Quere, Capillarity and Wetting Phenomena: Drops, Bubbles, Pearls, Waves (Springer, New York, 2004).

[3] T. Huhtamäki, X. Tian, J. T. Korhonen, and R. H. A. Ras, Surface-Wetting Characterization Using Contact-Angle Measurements, Nat. Protocols 13, 1521 (2018).

[4] C. W. Extrand and Y. Kumagai, Contact Angles and Hysteresis on Soft Surfaces, J. Colloid Interface Sci. 184, 191 (1996).

[5] T. Kajiya, A. Daerr, T. Narita, L. Royon, F. Lequeux, and L. Limat, Advancing Liquid Contact Line on Visco-Elastic Gel Substrates: Stick-Slip vs. Continuous Motions, Soft Matter 9, 454 (2013).

[6] A. Carre, J.-C. Gastel, and M. E. R. Shanahan, Viscoelastic Effects in the Spreading of Liquids, Nature (London) 379, 432 (1996).

[7] R. Pericet-Camara, A. Best, H.-J. Butt, and E. Bonaccurso, Effect of Capillary Pressure and Surface Tension on the Deformation of Elastic Surfaces by Sessile Liquid Microdrops: An Experimental Investigation, Langmuir 24, 10565 (2008).

[8] E. R. Jerison, Y. Xu, L. A. Wilen, and E. R. Dufresne, Deformation of an Elastic Substrate by a Three-Phase Contact Line, Phys. Rev. Lett. 106, 186103 (2011).

[9] R. W. Style, R. Boltyanskiy, Y. Che, J. S. Wettlaufer, L. A. Wilen, and E. R. Dufresne, Universal Deformation of Soft Substrates Near a Contact Line and the Direct Measurement of Solid Surface Stresses, Phys. Rev. Lett. 110, 066103 (2013).

[10] M. Van Gorcum, S. Karpitschka, B. Andreotti, and J. Snoeijer, Spreading on Viscoelastic Solids: Are Contact Angles Selected by Neumann's Law?, Soft Matter 16, 1306 (2020).

[11] Q. Xu, K. E. Jensen, R. Boltyanskiy, R. Sarfati, R. W. Style, and E. R. Dufresne, Direct Measurement of Strain-Dependent Solid Surface Stress, Nat. Commun. 8, 555 (2017).

[12] F. Boulogne, F. Ingremeau, L. Limat, and H. A. Stone, Tuning the Receding Contact Angle on Hydrogels by Addition of Particles, Langmuir 32, 5573 (2016). 
[13] F. J. Holly and M. F. Refojo, Water Wettability of Hydrogels, Hydrogels for Medical and Related Applications (American Chemical Society, Washington D.C., 1976), Chap. 19, pp. 252-266.

[14] X. Yao, L. Chen, J. Ju, C. Li, Y. Tian, L. Jiang, and M. Liu, Superhydrophobic Diffusion Barriers for Hydrogels via Confined Interfacial Modification, Adv. Mater. 28, 7383 (2016).

[15] Y. A. Meier, K. Zhang, N. D. Spencer, and R. Simic, Linking Friction and Surface Properties of Hydrogels Molded Against Materials of Different Surface Energies, Langmuir 35, 15805 (2019).

[16] H. Yasuda, A. K. Sharma, and T. Yasuda, Effect of Orientation and Mobility of Polymer Molecules at Surfaces on Contact Angle and Its Hysteresis, J. Polym. Sci. 19, 1285 (1981).

[17] R. Bai, J. Yang, and Z. Suo, Fatigue of Hydrogels, Eur. J. Mech. A Solids 74, 337 (2019).

[18] C. Monteux, A. Tay, T. Narita, Y. De Wilde, and F. Lequeux, The Role of Hydration in the Wetting of a Soluble Polymer, Soft Matter 5, 3713 (2009).

[19] D. Paretkar, X. Xu, C.-Y. Hui, and A. Jagota, Flattening of a Patterned Compliant Solid by Surface Stress, Soft Matter 10, 4084 (2014).

[20] Y. Hu, X. Zhao, J. J. Vlassak, and Z. Suo, Using Indentation to Characterize the Poroelasticity of Gels, Appl. Phys. Lett. 96, 121904 (2010).

[21] J. Yoon, S. Cai, Z. Suo, and R. C. Hayward, Poroelastic Swelling Kinetics of Thin Hydrogel Layers: Comparison of Theory and Experiment, Soft Matter 6, 6004 (2010).

[22] R. W. Style, A. Jagota, C.-Y. Hui, and E. R. Dufresne, Elastocapillarity: Surface Tension and the Mechanics of Soft Solids, Annu. Rev. Condens. Matter Phys. 8, 99 (2017).

[23] Q. Xu, R. W. Style, and E. R. Dufresne, Surface Elastic Constants of a Soft Solid, Soft Matter 14, 916 (2018).

[24] B. Andreotti and J. H. Snoeijer, Statics and Dynamics of Soft Wetting, Annu. Rev. Fluid Mech. 52, 285 (2020).

[25] R. Boltyanskiy, J. W. Merrill, and E. R. Dufresne, Tracking Particles with Large Displacements Using Energy Minimization, Soft Matter 13, 2201 (2017).

[26] See Supplemental Material at http://link.aps.org/ supplemental/10.1103/PhysRevX.11.031004 for analytic expressions for $U_{\perp}$ and $U_{\|}$and further experimental data.

[27] Y. Xu, W. C. Engl, E. R. Jerison, K. J. Wallenstein, C. Hyland, L. A. Wilen, and E. R. Dufresne, Imaging In-Plane and Normal Stresses Near an Interface Crack Using Traction Force Microscopy, Proc. Natl. Acad. Sci. U.S.A. 107, 14964 (2010).

[28] O. I. Del Rio and A. W. Neumann, Axisymmetric Drop Shape Analysis: Computational Methods for the Measurement of Interfacial Properties from the Shape and Dimensions of Pendant and Sessile Drops, J. Colloid Interface Sci. 196, 136 (1997).

[29] O. Ronsin, C. Caroli, and T. Baumberger, Preferential Hydration Fully Controls the Renaturation Dynamics of Collagen in Water-Glycerol Solvents, Eur. Phys. J. E 40, 55 (2017).

[30] S. Mora, T. Phou, J.-M. Fromental, L. M. Pismen, and Y. Pomeau, Capillarity Driven Instability of a Soft Solid, Phys. Rev. Lett. 105, 214301 (2010).
[31] A. Chakrabarti and M. K. Chaudhury, Direct Measurement of the Surface Tension of a Soft Elastic Hydrogel: Exploration of Elastocapillary Instability in Adhesion, Langmuir 29, 6926 (2013).

[32] R. D. Schulman, M. Trejo, T. Salez, E. Raphaël, and K. Dalnoki-Veress, Surface Energy of Strained Amorphous Solids, Nat. Commun. 9, 982 (2018).

[33] H. Liang, Z. Cao, Z. Wang, and A. V. Dobrynin, Surface Stress and Surface Tension in Polymeric Networks, ACS Macro Lett. 7, 116 (2018).

[34] R. Masurel, M. Roché, L. Limat, I. Ionescu, and J. Dervaux, Elastocapillary Ridge as a Noninteger Disclination, Phys. Rev. Lett. 122, 248004 (2019).

[35] R. Shuttleworth, The Surface Tension of Solids, Proc. Phys. Soc. London Sect. A 63, 444 (1950).

[36] H. Y. Erbil, G. McHale, S. M. Rowan, and M. I. Newton, Determination of the Receding Contact Angle of Sessile Drops on Polymer Surfaces by Evaporation, Langmuir 15, 7378 (1999).

[37] R. Lhermerout, H. Perrin, E. Rolley, B. Andreotti, and K. Davitt, A Moving Contact Line as a Rheometer for Nanometric Interfacial Layers, Nat. Commun. 7, 12545 (2016).

[38] D. Guan, E. Charlaix, and P. Tong, State and Rate Dependent Contact Line Dynamics over an Aging Soft Surface, Phys. Rev. Lett. 124, 188003 (2020).

[39] T. Baumberger, C. Caroli, and D. Martina, Solvent Control of Crack Dynamics in a Reversible Hydrogel, Nat. Mater. 5, 552 (2006).

[40] M. Grzelka, J. B. Bostwick, and K. E. Daniels, Capillary Fracture of Ultrasoft Gels: Variability and Delayed Nucleation, Soft Matter 13, 2962 (2017).

[41] F. J. Holly and M. F. Refojo, Wettability of Hydrogels I. Poly (2-Hydroxyethyl Methacrylate), J. Biomed. Mater. Res. 9, 315 (1975).

[42] H.-J. Butt, R. Berger, W. Steffen, D. Vollmer, and S. A. L. Weber, Adaptive Wetting-Adaptation in Wetting, Langmuir 34, 11292 (2018).

[43] J. H. Snoeijer, E. Rolley, and B. Andreotti, Paradox of Contact Angle Selection on Stretched Soft Solids, Phys. Rev. Lett. 121, 068003 (2018).

[44] M. van Gorcum, B. Andreotti, J. H. Snoeijer, and S. Karpitschka, Dynamic Solid Surface Tension Causes Droplet Pinning and Depinning, Phys. Rev. Lett. 121, 208003 (2018).

[45] S. Mora, M. Abkarian, H. Tabuteau, and Y. Pomeau, Surface Instability of Soft Solids under Strain, Soft Matter 7, 10612 (2011).

[46] X. Shao, S. A. Fredericks, J. R. Saylor, and J. B. Bostwick, A Method for Determining Surface Tension, Viscosity, and Elasticity of Gels via Ultrasonic Levitation of Gel Drops, J. Acoust. Soc. Am. 147, 2488 (2020).

[47] N. Nadermann, C.-Y. Hui, and A. Jagota, Solid Surface Tension Measured by a Liquid Drop under a Solid Film, Proc. Natl. Acad. Sci. U.S.A. 110, 10541 (2013).

[48] S. Mondal, M. Phukan, and A. Ghatak, Estimation of Solid-Liquid Interfacial Tension Using Curved Surface of a Soft Solid, Proc. Natl. Acad. Sci. U.S.A. 112, 12563 (2015).

[49] D. Chen, S. Cai, Z. Suo, and R. C. Hayward, Surface Energy as a Barrier to Creasing of Elastomer Films: An Elastic 
Analogy to Classical Nucleation, Phys. Rev. Lett. 109, 038001 (2012).

[50] R. W. Style and E. R. Dufresne, Static Wetting on Deformable Substrates, from Liquids to Soft Solids, Soft Matter 8 , 7177 (2012).

[51] J. B. Bostwick, M. Shearer, and K. E. Daniels, Elastocapillary Deformations on Partially-Wetting Substrates: Rival Contact-Line Models, Soft Matter 10, 7361 (2014).

[52] A. Bardall, K. E. Daniels, and M. Shearer, Deformation of an Elastic Substrate due to a Resting Sessile Droplet, Eur. J. Appl. Math. 29, 281 (2018).
[53] R. W. Style and Q. Xu, The Mechanical Equilibrium of Soft Solids with Surface Elasticity, Soft Matter 14, 4569 (2018).

[54] W. Gross and H. Kress, Simultaneous Measurement of the Young's Modulus and the Poisson Ratio of Thin Elastic Layers, Soft Matter 13, 1048 (2017).

[55] https://doi.org/10.5281/zenodo.4884194

[56] R. W. Style, R. Boltyanskiy, G. K. German, C. Hyland, C. W. MacMinn, A. F. Mertz, L. A. Wilen, Y. Xu, and E. R. Dufresne, Traction Force Microscopy in Physics and Biology, Soft Matter 10, 4047 (2014). 